\title{
Topical sirolimus solution for lingual microcystic lymphatic malformations in children and adults (TOPGUN): study protocol for a multicenter, randomized, assessor-blinded, controlled, stepped- wedge clinical trial
}

\section{Antoine MARCHAND ( $\square$ marchandtoine@gmail.com )}

Centre Hospitalier Régional Universitaire de Tours: Centre Hospitalier Regional Universitaire de Tours https://orcid.org/0000-0003-1858-8159

\section{Agnès CAILLE}

Centre Hospitalier Régional Universitaire de Tours: Centre Hospitalier Regional Universitaire de Tours Valérie GISSOT

Centre Hospitalier Régional Universitaire de Tours: Centre Hospitalier Regional Universitaire de Tours

\section{Bruno GIRAUDEAU}

Centre Hospitalier Régional Universitaire de Tours: Centre Hospitalier Regional Universitaire de Tours Céline LENGELLE

Centre Hospitalier Régional Universitaire de Tours: Centre Hospitalier Regional Universitaire de Tours Hélène BOURGOIN

Centre Hospitalier Régional Universitaire de Tours: Centre Hospitalier Regional Universitaire de Tours Bérenger LARGEAU

Centre Hospitalier Régional Universitaire de Tours: Centre Hospitalier Regional Universitaire de Tours Sophie LEDUCQ

Centre Hospitalier Régional Universitaire de Tours: Centre Hospitalier Regional Universitaire de Tours Annabel MARUANI

Centre Hospitalier Régional Universitaire de Tours: Centre Hospitalier Regional Universitaire de Tours

Study protocol

Keywords: Lingual Microcystic Lymphatic Malformation, Lymphangioma, Lymphatic Abnormalities, Sirolimus, Mucosa, Topical Treatment, Stepped-wedge Design

Posted Date: May 20th, 2021

DOI: https://doi.org/10.21203/rs.3.rs-515880/v1 
License: (c) (i) This work is licensed under a Creative Commons Attribution 4.0 International License. Read Full License 
1 Title: Topical sirolimus solution for lingual microcystic lymphatic malformations in children and

2 adults (TOPGUN): study protocol for a multicenter, randomized, assessor-blinded, controlled,

3 stepped-wedge clinical trial

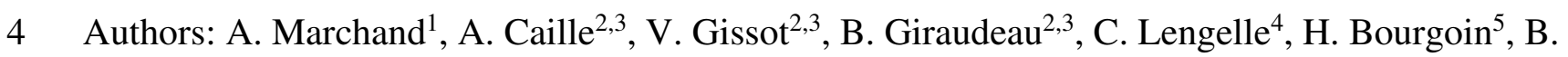

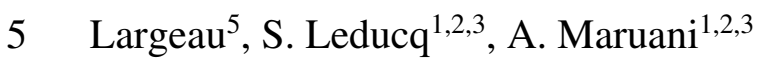

6 1: Department of Dermatology and Reference Center for Rare Diseases and Vascular Malformations

7 (MAGEC), CHRU Tours, Avenue de la République, 37044, Tours, Cedex 9, France.

8 marchandtoine@gmail.com, soleducq@gmail.com, annabel.maruani@univ-tours.fr

92 : Clinical Investigation Center, INSERM 1415, CHRU Tours, 37000, Tours, France.

10 agnes.caille@univ-tours.fr, valerie.gissot@univ-tours.fr, $\underline{\text { bruno.giraudeau@univ-tours.fr }}$

11 3: INSERM U1246 -SPHERE « MethodS in Patients-centered outcomes and HEalth REsearch »,

12 University of Nantes, University of Tours, 37000, Tours, France.

13 4: Pharmacovigilance Regional Centre (CRPV), CHRU Tours, 37000, Tours, France.

14 c.lengelle@chu-tours.fr

15 5: Department of Pharmacy, University Hospital Center of Tours, 37000, Tours, France.

16 h.bourgoin@chu-tours.fr, berenger.largeau@gmail.com

17 Corresponding authors: A. Marchand, A. Maruani 


\section{Abstract}

2 Background:

3 Lingual microcystic lymphatic malformations (LMLMs) are rare congenital vascular malformations

4 presenting as clusters of cysts filled with lymph fluid or blood. Even small well-limited lesions can be

5 responsible for a heavy burden, inducing pain, aesthetic prejudice, or oozing, bleeding, infections. The

6 natural history of LMLMs is progressive worsening punctuated by acute flares. Therapeutic options

7 include surgery, laser excision and radiofrequency ablation but all are potentially detrimental and

8 expose to local relapse. Therefore, the management frequently relies on a "watchful waiting"

9 approach. In complicated LMLMs, treatment with oral sirolimus, a mammalian target of rapamycin

10 (mTOR) inhibitor, is often used. Topical applications of sirolimus on the buccal mucosae have been

11 reported in other oral diseases with good tolerance and none to slight detectable blood sirolimus

12 concentrations. We aim to evaluate the efficacy and safety of a $1 \mathrm{mg} / \mathrm{mL}$ sirolimus solution applied

13 once daily on LMLM of any stage in children and adults after 4, 8, 12, 16, 20 and 24 weeks of

14 treatment compared to usual care (no treatment).

15 Methods:

16 This is a randomized, multicentric study using an individually randomized stepped-wedge design over

1724 weeks to evaluate topical application of a $1 \mathrm{mg} / \mathrm{mL}$ sirolimus solution once daily, on LMLM,

18 versus usual care (no treatment), the control condition. Participants begin with an observational period

19 and later switch to the intervention at a randomized time (week 0,48 or 12). Visits occur every 4

20 weeks, either in the study center or by teleconsulting. The primary outcome will be the evaluation of 
1 global severity of the LMLM on monthly standardized photographs by 3 independent blinded experts

2 using the physical global assessment (PGA) 0 to 5 scale. Secondary outcomes will include lesion size

3 measurement and quality of life assessment, investigator and patient-assessed global disease and

4 specific symptoms (oozing, bleeding, sialorrhea, eating impairment, taste modification, aesthetic

5 impairment, pain, and global discomfort) assessment. A biological monitoring will be performed

6 including residual blood sirolimus concentration and usual laboratory parameters.

7 Discussion:

8 Given the disappointing state of current treatment options in LMLMs, topical sirolimus could become

9 first-line therapy in treating LMLMs if its efficacy and safety were to be demonstrated.

11 Trial registration:

12 Clinicaltrials.gov : NCT04128722, registered $24^{\text {th }}$ September 2019,

13 https://clinicaltrials.gov/ct2/show/NCT04128722

14 EudraCT : EUCTR2019-001530-33-FR

15 Sponsor (University Hospital Center of Tours - CHRU Tours) : DR190041-TOPGUN

16 French regulatory authorities : ID RCB: 2019-001530-33 
1 Keywords: Lingual Microcystic Lymphatic Malformation, Lymphangioma, Lymphatic Abnormalities,

2 Sirolimus, Mucosa, Topical Treatment, Stepped-wedge Design

3

4 Abbrevation list

5 AKT Protein kinase B

6 ALAT Alanine AminoTransferase

$7 \quad$ ANSM

Agence Nationale de la Sûreté du Médicament (French national drug safety

8 agency)

9 ASAT Aspartate Aminotransferase

10 CIC-P Centre d'Investigation Clinique - Polyvalent (Clinical Investigation Center)

11 CPP Comité de Protection des Personnes (Ethics Committee)

12 cDLQI Children's Dermatologic Life Quality Index

13 DLQI Dermatologic Life Quality Index

14 DSMB Data Safety Monitoring Board

4 
$5 \quad$ Biomedical Research Agency)

6 ISSVA

7 LM

8 LMLM

9 mTOR

$10 \quad \mathrm{PI} 3 \mathrm{k}$

$11 \quad$ PGA

12 SOP

13 SUSAR

$14 \quad \operatorname{VEGF}(\mathrm{R})$
International Society for the Study of Vascular Anomalies

Lymphatic Malformation

Lingual Microcystic Lymphatic Malformation

Mammalian Target Of Rapamycin

Phosphoinositide 3-kinase

Physician's Global Assessment

Standardized Operating Procedures

Suspected Unexpected Serious Adverse Reaction

Vascular Endothelial Growth Factor (Receptor) 


\section{Background}

2 Lymphatic malformations (LMs) are rare congenital anomalies (estimated prevalence $<0.1 \%)^{1}$. They belong to

3 the wider group of vascular malformations, according to the International Society for the Study of Vascular

4 Anomalies (ISSVA) 2014 classification ${ }^{2,3}$, as part of low-flow vascular malformations. They can be isolated or

5 associated with other anomalies or birth defects such as Proteus syndrome or CLAPO syndrome. LMs present

6 as clusters of cysts filled with translucid fluid or blood that can be macrocystic, microcystic or mixed

7 type. Any part of the skin or mucosae can be involved, with or without involvement of the underlying

8 deep structures. At least $50 \%$ of LMs can be found in the head and neck area ${ }^{4}$. In this area, the buccal

9 cavity is the most often involved, especially in medial microcystic LMs of the tongue ${ }^{10,11}$. Despite their

10 very heterogenous clinical severity and presentations, all medial microcystic LMs are classified as

11 stage III according to de Serres et al., which implies high post-surgical relapse rate and peri-operative

12 complications.

13 Specifically, patients with LMLMs, even those with small well-limited lesions, often experience a

14 heavy burden because of oozing, spontaneous bleeding, infections, or even speech, chewing or

15 breathing impairment. An important aesthetic prejudice is also frequently reported ${ }^{12,13}$. The disease's

16 natural history features progressive worsening, often punctuated by acute flares such as infections,

17 bleeding, or acute swelling, sometimes secondary to lymphatic drainage-stimulating conditions such as

18 trauma or ear-nose-throat regional infections ${ }^{13}$. 
1 Current therapeutic options are still limited and mostly rely on surgery or local interventional

2 procedures. Although sclerotherapy may yield good results in macrocystic LMs, where large well-

3 delimited cysts, this standard treatment option remains disappointing in more ill-defined infiltrative

4 microcystic LMs, with high rates of post-surgical relapse or deep-structure iatrogenic lesions ${ }^{14-16}$.

5 Complete surgical resection is rarely achievable in the oral cavity and can be tied to significant post-

6 operative complications, such as post-operative oedema, post-operative infections, delayed healing or

7 facial nerve palsies in deep infiltrative oral $\mathrm{LMs}^{14}$. Multiple partial resections may achieve functional 8 improvement while minimizing drawbacks.

9 Other interventional techniques reported include CO2-laser excision or radiofrequency ablation, most

10 often for superficial LMs or as combination therapy after surgery for larger LMs. However, they often

11 lead to reduced symptoms but only very rarely achieve permanent curative results and may induce

12 deeper tissue scarring that might hamper secondary function-preserving surgery ${ }^{11}$.

13 Thus, LMLM management remains challenging, and a conservative management ("wait-and-see"

14 approach) is frequently chosen. Iterative courses of antibiotics and steroids are started in response to

15 acute flares or infections, with the caveat that infection-induced LMLM growth may render secondary

16 ablation difficult.

17 The mammalian target of rapamycin (mTOR) is an intracellular kinase downstream from the PI3K-

18 AKT pathway. When activated by the effect of the PI3K-AKT complex, it binds with other 
1 intracellular proteins, forming serine-threonine kinase mTOR complexes 1 and 2, which are involved

2 in cell growth and apoptosis via protein synthesis and cell-cycle regulation ${ }^{17}$. The PI3K-AKT-mTOR

3 pathway is heavily involved during angiogenesis and lymphangiogenesis ${ }^{18}$.

4 Known mutations in the PI3K-AKT-mTOR pathway have been discovered in both non-syndromic and

5 syndromic lymphatic abnormalities, such as Proteus syndrome, Pi3K-related overgrowth spectrum-

6 linked diseases (CLOVES syndrome or Klippel-Trenaunay syndrome) $)^{5,19,20}$.

7 Sirolimus is a powerful inhibitor of the mTOR kinase, thereby preventing cellular growth and leading

8 to apoptosis. It has been granted US Food and Drug Administration approval for preventing chronic

9 allograft rejection in renal transplant recipient. In vitro, applying sirolimus to rat pancreas cancer-

10 derived cells reduced VEGF-C expression and inhibited lymphangiogenesis; similar results were found

11 in mice inoculated with the cells ${ }^{18}$. Similarly, sirolimus-treated cells from LMs showed inhibited

12 growth ${ }^{19}$, whether cultivated from syndromic (Proteus syndrome) or isolated LMs.

13 The first oral sirolimus administration as a compassionate treatment in refractory LMs was reported in

142008 in a 9-month-old boy ${ }^{23}$ with multiorgan failure due to Proteus syndrome. Since then, sirolimus

15 has been applied in different vascular anomalies ${ }^{13,24}$, with 2 RCTs of LMs currently under way

16 (NCT00975819, Adams, United States [active] and NCT02509468, Maruani, France [completed] $)^{25}$.

17 However, despite an overall good safety profile (absent or mild side effects in 2 of 3 patients with

18 vascular anomalies, including children), significant dermatologic (oral mucositis), hepatic (elevated 
1 liver enzymes, hepatitis) or hematologic side effects may occur. Grade III or IV drug-induced

2 cytopenia is reported in up to $27 \%$ of treated patients, with some severe infectious complications also

3 being reported ${ }^{13,24}$.

4 Hence, close clinical and biological follow-up is required, with sirolimus blood concentration

5 monitoring. Treatment indications tend to be limited to the most severe lesions (i.e., extensive deep

6 infiltration, aerodigestive airway involvement).

7 Topical sirolimus on skin and mucosae:

8 The first topical use of sirolimus was reported in a 2005 pilot study involving 24 patients with plaque

9 psoriasis $^{26}$. It has also been tried in multiple inflammatory or vascular-based skin and mucosae

10 diseases such as oral erosive lichen planus $^{27}$, oral pemphigus vulgaris ${ }^{28}$, port-wine stains or tuberous

11 sclerosis-induced facial angiofibromas ${ }^{29,30,31}$.

12 There are few case reports describing sirolimus use in cutaneous microcystic LMs, in both children

13 and adults, yielding promising results regarding lesion shrinking and a sharp reduction in the

14 frequency of acute bleeding, swelling or infectious episodes ${ }^{32,33}$.

15 Mucosal topical sirolimus treatment has been reported in some inflammatory conditions. Undiluted

16 commercial oral sirolimus solution (Rapamune ${ }^{\circledR}$, Pfizer) had moderate effect in seven females with

17 oral and/or genital lichen planus ${ }^{27}$. The tolerance was overall good, with only one treatment

18 interruption due to local irritation. Blood sirolimus levels were monitored, with a peak sirolimus 9 
1 concentration of $1.5 \mathrm{ng} / \mathrm{mL}$, well below usual therapeutic concentrations of 4 to $12 \mathrm{ng} / \mathrm{mL}$, in only one

2 patient with both oral and genital involvement, $2 \mathrm{hr}$. after treatment.

3 Nudelmann et al. ${ }^{34}$ performed more extensive studies of oral sirolimus as a $0.05 \%$ mouthwash.

4 Therapeutic sirolimus concentrations were detected in saliva up to $4 \mathrm{hr}$. after mouth washing, and peak

5 blood sirolimus concentrations were from 0.2 to $1 \mathrm{ng} / \mathrm{mL}$ at $1 \mathrm{hr}$. after a single rinse with sirolimus

6 mouthwash. No immediate side effects were reported, and no significant change was reported between

7 an oral cavity examination performed by an oral medicine specialist before and $24 \mathrm{hr}$. after using the

8 mouthwash.

9 Objectives

10 Hence, we aim to perform a clinical trial (TOPical sirolimus in linGUal microcystic lymphatic

11 malformatioN [TOPGUN]) to assess the efficacy and safety of a $1-\mathrm{mg} / \mathrm{mL}$ sirolimus solution applied

12 once daily as a topical treatment for oral microcystic LMs with lingual involvement in children and

13 adults compared to usual care (no treatment).

14 Methods

15 A. Trial design

16 TOPGUN is a randomized, open, multicenter study with an individually randomized stepped-wedge

17 design ${ }^{35}$ over a 24 -week period to evaluate the following:

10 
- daily topical application of $1 \mathrm{mg} / \mathrm{mL}$ sirolimus solution 0.5 to $1 \mathrm{~mL}$ according to the size of the LMLM lesion, the experimental intervention, versus

- usual care (no treatment), the control condition

4 In this design, participants are included in a cohort in which treatment is introduced at a randomized

5 timepoint. Included participants start the intervention in a staggered fashion over time. Randomization

6 is balanced, so each participant has the same probability of starting the intervention at a given step as

7 any other participant over the entire study period. Four steps are planned for starting the intervention

8 (i.e., Week 0 [W0], W4, W8 and W12). Hence, 3 participants will be randomly assigned to begin the

9 intervention on W0, 3 on W4, 3 on W8 and 3 on W12.

10 All patients will undergo a visit (physical, "on-the-spot" visit or via secured phoning software or phone

11 calls, according to the step-specific participant timeline), every 4 weeks until W24, when the

12 intervention will be stopped. Assessments before starting the intervention will be compared to those

13 after the introduction of the intervention.

14 The individually randomized stepped-wedge trial design is increasingly being used in clinical practice,

15 often in rare diseases for which patient recruitment may be difficult. Because of this very rare disease,

16 randomizing the first topical sirolimus-treated LMLM patients seemed important to obtain high-quality

17 evidence.

18 Other advantages of the stepped-wedge approach include the following ${ }^{35,36}$ : 
- Each patient is his own control, hence increasing trial power with a small patient count, while taking into account a possible persisting treatment effect (contrary to cross-over designs)

- Being able to evaluate the effect of the time of treatment introduction and treatment duration owing to the varying treatment courses from 12 to 24 weeks.

- Increasing trial acceptability in this rare disease. Indeed, during the study, participants need to comply with a significantly tighter clinical follow-up than during usual care and might be reluctant to apply for trial inclusion if they believe they will not receive the experimental treatment, especially if they live far from the study center. Resorting to phone calls instead of traditional face-to-face visits also aims to address this matter.

We could not use a placebo drug because of pharmaceutical stability issues. As a result, neither the patient nor the investigator is blinded to the treatment introduction timepoint. However, the main outcome will be assessed on photographs by an independent blinded adjudication committee.

\section{B. Methods: Participants, interventions, and outcomes}

1. Study setting

The study will involve 3 French hospital centers (University Hospital of Tours, Regional Hospital of Orléans and hospital Necker-Enfants maladies, Paris) that are currently involved in the treatment of vascular anomalies.

\section{Eligibility criteria}


a) Inclusion

2 Eligible patients will be at least 5 years old. They must have a diagnosis of LMLM of any stage

3 (Wiegand 2009) ${ }^{11}$, assessed by clinical examination, with or without an underlying syndromic

4 malformation (e.g., Proteus or CLAPO syndrome). Deep infiltration must have been assessed by head-

5 and-neck MRI before study enrolment.

6 We chose a 5-year age threshold because although LMLMs are often diagnosed before age 2 years,

7 they might not be clinically relevant in very young children, given their natural history of progressive

8 worsening. Also, the topical administration that requires not swallowing the oral solution might be

9 difficult in younger children with smaller oral cavities. Finally, in this study, we can use validated

10 quality-of-life assessment instruments (Children's Dermatologic Life Quality Index [cDLQI]), which

11 cannot be used for children under age 4 years ${ }^{37}$.

b) Exclusion

13 Patients with any of the following conditions are not included in the study:

14 - lymphatic malformation that requires a continued background therapy (involving deep organs)

15 - secondary lymphatic malformations (e.g., radiotherapy-induced lymphangiectasia)

16 - immunosuppression (immunosuppressive disease or immunosuppressive treatment)

$17 \quad$ - ongoing neoplasia

18 - active chronic infectious disease (e.g., hepatitis B and C virus, HIV)

19 - Local necrosis

13 
- Local fungal, viral (herpes simplex virus, varicella-zoster virus, etc.) or bacterial infection on the site of the LMLM (based on clinical examination)

- Previous treatment with systemic or topical mTOR inhibitors within 6 months before inclusion (oral sirolimus half-life is $60 \mathrm{hr}$ in adults according to Summary of Product Characteristics for

- Previous treatment with oral or topical steroids within 10 days before inclusion (half-life of corticosteroids is $12-36 \mathrm{hr}$.)

$9 \quad-\quad$ Soybean or peanut allergy

- Pregnant or breastfeeding

11 - Female patients of child-bearing potential not using a reliable contraceptive method

14 During the observation (control) period will receive usual care for their LMLM, which is no treatment 15 at this stage of the condition.

16 Then, after crossing over to the experimental period, the intervention is the $1 \mathrm{mg} / \mathrm{mL}$ sirolimus oral 17 solution (Rapamune ${ }^{\circledR}$, Pfizer), administered once daily. If the LMLM covers below an estimated $<4$ $18 \mathrm{~cm}^{2}$ of target area, the treatment dose is $0.5 \mathrm{~mL}$, once daily. If the LMLM covers above an estimated $19 \geq 4 \mathrm{~cm}^{2}$ of target area, the treatment dose is $1 \mathrm{~mL}$, once daily. 
1 A total of 0.5 or $1 \mathrm{~mL}$ non-diluted sirolimus solution will be taken from the bottle by using the 1-use

2 dosing syringe and applied on a standardized disposable applicator device. Then the applicator device

3 will be gently applied, only on the LMLM, until the solution is no longer oozing from the applicator

4 device, for at least $10 \mathrm{sec}$. The patient must then not eat, drink, brush teeth, or use mouthwash for $1 \mathrm{hr}$.

5 Applicators and syringes are to be disposed of adequately. Rapamune ${ }^{\circledR}$ bottles must be brought at

6 every dispensation visit to assess patient observance. Syringes must not be re-used.

7 The treatment will be started at a randomized timepoint (W0, W4, W8 or W12) and will be taken until

8 W24 whatever the starting time.

9 In case of serious adverse reactions, including local necrosis or any systemic reaction, the intervention

10 (topical sirolimus) will be stopped. In case of local side effects, the intervention might be temporarily

11 halted. If the patient, the sponsor and the investigator deem it appropriate, the intervention can be

12 resumed, at full or half-dose. In case of relapse, the lowest bearable dose will be sought; otherwise, the

13 treatment might be stopped. If the lesion is totally removed before W24, the investigator could propose

14 early withdrawal of the treatment. In this setting, follow-up would be according to the protocol until

15 W24.

16 Participants (or together with their parents if they are < 18 years old) will be asked to keep a daily

17 participant diary, recording information about adherence and safety data. They will be asked to bring 
1 the diary at each study visit. At each on-site study visit, participants will be asked to provide all their

2 used and unused study drug containers.

3 Regarding concomitant care, in the event of an acute flare or local or generalized infection, antibiotics

4 may be prescribed. Systemic steroid therapy may also be prescribed along with antibiotics, for up to 3

5 days. However, topical immunosuppressive drugs or steroids applied on the target area, interventional

6 procedures (sclerotherapy, laser, radiofrequency) or surgery of the target area, and systemic steroids

7 for more than 3 days, or immunosuppressive therapy, including systemic mTOR inhibitors, are

8 prohibited during the study. If any of the above is administered, then the investigator must be

9 informed, and the intervention must be stopped. In any case, all assessments planned should be 10 performed.

4. Outcomes

a) Primary outcome

13 The primary outcome will be the evaluation of global severity of the LMLM by 3 independent experts

14 (i.e., adjudication committee). To this end, the experts will compare the LMLM by using a 6-point

15 physician's global assessment (PGA) scale. The PGA score ranges from 0 (clear) to 5 (severe).

16 Photographs will be taken at baseline and every 4 weeks up to W24 by using a standardized

17 photograph protocol (3 photos: 2 front photos [distances 8-10 and 25-27 cm] and 1 side photo

18 [distance 8-10 cm]). Patients (and their families if relevant) will be trained in the photograph protocol 16 
1 during the inclusion visit. During on-the-spot visits in the study centre, photographs will be taken by a

2 trained research nurse or investigator. When at home, photographs will be taken by the patient

3 themselves or their family members and sent to a secured email address. The quality of the

4 photographs will be controlled immediately by the investigator, and the photograph protocol might be

5 resumed until the quality is deemed satisfactory. Then, the photographs will be immediately

6 anonymized.

7 The adjudication committee will be blinded to treatment allocation. They will quantify disease severity

8 for each photograph by using the PGA scale.

9 There are no specific scores for LMLMs. The only existing score for head-and-neck lymphatic

10 malformations is the Cologne Disease Score ${ }^{12}$, but it focuses on disease induced morbidity, with no

11 morphology-related item, and some of its items (feeding impairment, respiration impairment) may

12 apply to more severe lesions we do not aim to investigate. However, the PGA score is an easy-to-

13 understand and widely used instrument in dermatology $y^{38,39}$. We believe it relevant for this condition.

14 The PGA score ranges from 0 (clear) to 5 (severe) A 1-point improvement versus baseline in PGA

15 scale would already be of clinical relevance.

b) Secondary outcomes

17 Secondary outcomes will assess topical sirolimus efficacy and safety. They include investigator- and

18 patient-reported outcomes, with an emphasis on quality of life and symptoms.

19 - Investigator-assessed PGA at weeks $0,4,8,12,16,20$ and 24

17 
1 - Patient-assessed oozing, bleeding, sialorrhea, eating impairment, taste modification, aesthetic

2 impairment, pain and global discomfort, each on a numeric scale from 0 to 10 ( 0 , clear; 10 , very

3 severe), at weeks $0,4,8,12,16,20$ and 24

4 - Global evolution assessed by the patient from -10 to 10 (-10, severe worsening; 0 , no change; 10 ,

5 complete recovery), at weeks $4,8,12,16,20$ and 24

6 - Quality of life assessment (cDLQI for patients 5 to 16 years or DLQI > 16 years), at baseline,

7 treatment introduction visit and W24

8 - Measurement of the lesion (length, width, thickness) by the investigator, at baseline, treatment

9 introduction visit and W24.

10 - Time to obtain optimal results (i.e., time from treatment introduction visit to time reaching the

11 minimal PGA score)

12 Reported side effects with oral sirolimus treatment include fatigue, headache, oral mucositis,

13 hypertension, dyslipidemia, hyperglycemia, cytopenia and infections [nadal2016]. Topical application

14 of sirolimus in oral diseases has also been tied to local irritation and pain [Soria2009]. Hence, to

15 account for these reported adverse effects, safety assessments will be both clinical and biological. The

16 clinical surveillance will include the recording of clinical local and general side effects according to

17 the daily completion of the patient follow-up diary, questions from the investigator at each visit and

18 physical examination and blood pressure measurement at each on-the-spot visit. Biological follow-up

19 will include residual sirolimus blood-level monitoring (after 4 weeks of treatment, then 8 weeks, then 18 
every 8 weeks until W24) and biological safety assessments at W8, W16 and W24 of exposure, compared to baseline. Although we do not expect an important systemic diffusion, the biological surveillance measurements will mimic those used in usual systemic sirolimus treatment monitoring: complete blood count, liver (ASAT, ALAT, GGT) and renal (serum creatinine) function, lipids [triglycerides and cholesterol (i.e., total cholesterol, high-density lipoprotein cholesterol and lowdensity lipoprotein cholesterol estimated according to Friedewald's formula)] and glycaemia.

5. Participant timeline

The duration of participation is 24 weeks for each patient, whatever the step they are randomized in.

The participant timeline encompasses general and step-specific schedules, which are described in Table 1-4.
(1)
6. Sample size

We could not define a reasonable effect-size hypothesis because LMLM is a very rare disease, with currently no described pharmaceutical treatment for LMLM. Hence, we did not perform any sample size calculation.

Both children $>5$ years old and adults are recruited alike in TOPGUN because LMLMs can be found at every age. The University hospital of Tours is a tertiary reference center for cutaneous vascular anomaly care, which all investigators are involved in. We also reached out to other university hospital 
1 centers in the "Grand-Ouest" region in France and the Necker-Enfants Malades pediatric hospital,

2 located in Paris (authorization granted in January 2021).

3 Methods: Assignment of interventions

1. Allocation

a) Sequence generation and allocation concealment mechanism

6 Time of cross-over to the intervention will be randomly assigned to the patient at W0, W4, W8 or W12

7 with a 1:1:1:1 ratio allocation as per a computer (SAS-based)-generated randomization schedule.

8 Participants will be randomized by using Ennov Clinical, an online central randomization procedure.

9 To ensure allocation concealment, randomization will not be possible until the participant has been

10 recruited into the trial, especially with all selection criteria collected and met.

b) Implementation

12 The allocation sequence will be generated by a statistician not involved in the recruitment or follow-up 13 of participants.

15 We could not use a placebo drug because of pharmaceutical stability issues. Sirolimus oral solution is

16 stable for $<30$ days after opening. A recondition in unlabeled study bottles, which would be the sine 
qua non condition to consider a placebo drug, would have required very frequent treatment deliveries, which did not fit with our monthly stepped-wedge design and would have sharply increased study costs. As a result, neither the investigators nor participants will be blinded and there will be no placebo intervention. Both the patient and the investigator will be aware of his/her allocated time of cross-over to intervention. However, the adjudication committee, which will assess the primary outcome, will be blinded to treatment allocation by the use of standardized anonymized photographs.

\section{Methods: Data collection, management, and analysis}

9 LMLMs are a rare skin disease for which neither evaluation guidelines nor purposely developed evaluation instruments exist. The PGA scale is a tool widely used in dermatology. It represents a

11 global evaluation of disease severity. We use a 6-point PGA scale: 0, clear; 1, almost clear; 2, mild; 3,

12 moderate; 4, severe; 5 , very severe. The PGA scale is widely used in psoriasis but is also used in a 13 wide array of skin diseases ${ }^{38,39}$ and as an easily obtainable global evaluation of disease severity is an 14 interesting tool. The DLQI and cDLQI are widely used instruments to assess health-related quality of 15 life in dermatology. The cDLQI is validated for children from age 4 years up to 16 years ${ }^{36}$.

16 Study staff with their own access right to the study database will enter/capture data from source 17 documents corresponding to a participant into the protocol-specific electronic Case Report Form 18 (eCRF). All information required by the protocol will be entered in the eCRF, and an explanation will 19 be provided for each missing piece of information. The data must be collected as they are obtained and 
1 transcribed into these forms in a clear manner. If a correction is required for an eCRF, the time and

2 date stamps track the person entering or updating eCRF data and create an electronic audit trail.

2. Data management

4 Data management will be performed by the INSERM CIC-P 1415. An eCRF will be developed by

5 using the Ennov Clinical software. eCRF management will be performed in agreement with the

6 INSERM CIC-P 1415 Standardized Operating Procedures (SOP). The Clinical Research Associate in

7 charge of the study will be trained in the eCRF and will be in charge of the investigator training. Data

8 will be entered in investigating centers via a secure web site, monitored by Clinical Research

9 Associates and potential queries will be edited by data managers, in agreement with a prespecified data 10 management plan.

11 Data will be reviewed before the database is locked. The database will be locked in agreement with the INSERM CIC-P 1415 SOPs, and data will be extracted in a SAS or other format according to

13 statistical requirements.

15 A detailed analysis plan will be a priori defined. SAS 9.4 and R 3.3.1 (or latest versions) will be used 16 for analysis. The level of statistical significance will be set at $5 \%$. 
1 The primary outcome (PGA) will be treated as a continuous variable. For analysis, we will use the

2 model described by Hooper et $\mathrm{al}^{36}$. Analysis will rely on a mixed linear regression model with a

3 random effect for participant. For each participant, we will include the 7 PGA measurements from W0

4 to W24 (assessed every 4 weeks).

5 The model will be as follows, denoting $Y_{i j t}$ as the PGA for individual $\mathrm{i}=1, . ., \mathrm{n}$ in sequence $\mathrm{j}=1, \ldots, \mathrm{T}$ at

6 time $\mathrm{t}=0, \ldots, \mathrm{T}$ after randomization:

$$
Y_{i j t}=\beta+\tau_{t}+A_{j t}+\eta_{i j}+\varepsilon_{i j t}
$$

8 where $\eta_{i j} \sim N\left(0, \sigma_{b}^{2}\right)$ is the participant random effect; $\varepsilon_{i j t} \sim N\left(0, \sigma_{w}^{2}\right)$ is the random error; and $\eta_{i j}$ and

$9 \varepsilon_{i j t}$ are all independent, and,

10

$$
A_{j t}\left\{\begin{array}{c}
0 \text { if sequence } j \text { is in the control condition at time } t \\
\alpha_{k} \text { if sequence } j \text { crosses to the intervention between times } t-k \text { and } t-k+1, k \geq 1
\end{array}\right.
$$

11 The mean of PGA for an individual participant follows a trajectory with baseline $\beta$ (mean PGA at

12 baseline) and time effects $\tau_{t}, \mathrm{t}=0, \ldots, 6$ with $\tau_{0}=0$.

13 When the participant crosses over to topical sirolimus, additional effects of treatment will be estimated

14 with $\alpha_{1}$ additional treatment effect in the first 4-week period after crossing over, changing to $\alpha_{2}, \alpha_{3}, \ldots$,

$15 \alpha_{6}$, mean additional treatment effect at W8, W12, W16, W20 and W24 after the introduction of

16 sirolimus respectively. 
1 This trial has a 4-sequence design, and our primary treatment effect of interest will be $\alpha_{3}$, the mean

2 difference in PGA at 12 weeks after the introduction of topical sirolimus. We will also estimate $\alpha_{1}, \alpha_{2}$,

$3 \alpha_{4}, \alpha_{5}$ and $\alpha_{6}$, mean additional treatment effects at W4, W8, W16, W20 and W24, respectively, after the 4 introduction of sirolimus.

$5 \quad$ Missing data will not be imputed for this analysis.

$6 \quad$ Individual trajectories of PGA will be plotted by using a spaghetti plot.

7 Secondary outcomes (global severity of disease and functional impairments) will be treated as

8 continuous variables ranging from 0 to 10 . We will use the same model as for the primary outcome.

9 Time to optimal results will be described with median and interquartile range. Safety data will be 10 reported using descriptive analysis.

\section{E. Methods: Monitoring}

13 A clinical research technician will be responsible for logistics of the study, producing reports

14 concerning its state of progress, ensuring eCRF completion and update (request for additional

15 information, corrections, etc.), and reporting severe adverse events to the sponsor. The person will

16 work in accordance with the SOP. 
1 A Data Safety Monitoring Board (DSMB) will consist of 2 dermatologists and 1 pharmacologist. The

2 DSMB is an advisory committee that discusses the benefit/risk ratio of the study and the

3 implementation of a clinical trial with the sponsor and the coordinating investigator of the study. The

4 board will be systematically questioned, at any time by the sponsor for each case of suspected

5 unexpected serious adverse reaction (SUSAR), once a year, before sending the safety report to French

6 Health Authorities and if data may change the benefit and risk ratio during the clinical trial.

7 At any time, the sponsor may refer to the DSMB to adjudicate whether an event is an SUSAR or a

8 severe adverse event when it is difficult to analyze or if new data change the benefit and risk ratio

9 during the clinical trial. The DSMB will analyze the transmitted data and may request additional

10 information and will make recommendations about the future of the clinical trial (continuation,

11 amendments, termination).

12 A clinical research associate appointed by the sponsor will regularly visit each study center according 13 to the monitoring plan depending on the frequency of inclusions and at the end of the study. During 14 these visits, informed consent, compliance with the study protocol, quality of the data collected in the 15 eCRF will be reviewed.

\section{Harms}

17 All serious and non-serious events will be reported on the adverse event reporting form (initial or

18 follow-up declaration), as thoroughly as possible, within the regulatory time limits for reporting. All

19 adverse events will be monitored until they are completely resolved. The investigator will immediately 25 
notify the sponsor of any serious adverse event. SUSARs will be reported to Eudravigilance (European

2 pharmacovigilance database), the French Health Authorities (ANSM), and the investigators.

\section{Auditing}

4 An audit may be performed at any time by people appointed by the sponsor who are independent of

5 those responsible for the study. The audit aims to ensure the good quality of the study and that the law

6 and regulations in force are being observed. The investigators agree to comply with the requirements

7 of the sponsor and the relevant authority for an audit or an inspection of the study.

8 The audit can apply to all stages of the study, from development of the protocol to publication of the

9 results and filing the data used or produced in the study.

10 F. Ethics and dissemination

12 The sponsor and the investigator or investigators undertake to conduct this study in compliance with

13 French law in force (Code de Santé Publique), the recommendations of French and international Good

14 Clinical Practices, the Helsinki Declaration (Ethical Principles for Medical Research involving Human

15 Subjects), and the European regulations related to clinical research. The study will be conducted in

16 accordance with this protocol. With the exclusion of emergency situations requiring specific

17 therapeutic actions, the investigators undertake to observe the protocol in all respects. 
1 This research is registered in the European EudraCT database in accordance with article L1121.15 of

2 the French Public Health Act.

3 This protocol has been granted both ANSM (French Regulatory Authority) and CPP (Comité de

$4 \quad$ Protection des Personnes, French Ethical Review Committee) approval (19.03.28.46025).

5 Copy of the ethics committee agreement is found in additional file 1.

7 Important protocol modifications will be submitted for approval to the institutional review board of the

8 University Hospital of Tours and will be communicated to coinvestigators.

9 The protocol was amended on December 12, 2019. Inclusion criteria were modified to LMLM of any

10 stage with lingual involvement, provided sirolimus is only applied on the anterior part of the tongue

11 (before the vallate papillae). The exclusion criterion "previous use of systemic sirolimus in the last 12

12 months" was modified to "previous use of systemic sirolimus in the last 6 months", which is still well

13 above a 7 half-life (i.e., $420 \mathrm{hr}$. = 3 weeks) clearance threshold. The intervention was not modified.

14 The aim of the amendment was to make screening easier in this very rare disease.

15 A second amendment has been authorized on January 22, 2021. It adds the Necker-Enfants-Malades

16 Hospital as a study center and extends the recruitment period by 1 year.

\section{Consent or assent}


1 The patient will be orally informed of the aim of the protocol and its procedures. Patients will also

2 receive a written information document. The written and informed consent of the patient, if obtained,

3 must be dated and signed both by the patient (or their parent or legal representatives). and the

4 investigator before any further study intervention. Children $\geq 16$ years old must also consent to use of

5 their data. The patient will receive a copy of the signed written consent and information letter. The

6 original Information Letter and Consent Form will be kept by the investigator (even if the patient

7 moves to a new hospital during the study) in a safe place inaccessible to third parties. The consent

8 form will be signed before any intervention needed for the study.

\section{Confidentiality}

10 In accordance with the legislative provisions in force (articles L.1121-3 and R.5121-13 of the French

11 Public Health Code), people with direct access to source data will take all necessary precautions to 12 ensure the confidentiality of information relating to the study intervention, research studies and people 13 taking part in them, particularly in regard to their identity and the results obtained. During the study or 14 when it is over, the information collected on the people taking part in it and forwarded to the sponsor 15 by the investigators (or any other specialized staff member involved) will be made anonymous. Under 16 no circumstances must the uncoded names or addresses of the people concerned appear in it.

18 The investigator will prepare and maintain adequate and accurate source documents designed to record 19 all observations and other pertinent data for each participant of the study. The sponsor is responsible 
1 for obtaining the agreement of all the parties involved in the study in order to guarantee direct access

2 in all the sites where the study is being conducted to source data, source documents and reports, so that

3 he/she can control their quality and audit them.

5 Any written or oral communication of the results of the study will be previously agreed by the

6 coordinating investigator and, if necessary, by the scientific committee constituted for the study.

7 Publication of the main results will mention the sponsor and the funding source. We will follow the

8 Recommendations for the Conduct, Reporting, Editing, and Publication of Scholarly Work in Medical

9 Journals (updated in December 2015) from the International Committee of Medical Journal Editors.

10 All investigators who are not cited in the authorship will be listed as non-author contributors. In

11 accordance with the law no. 2002-303 of March 4, 2002, participants will be informed, at their request, 12 of the overall results of the study.

\section{SPIRIT statement}

14 This protocol has been written in accordance with the Standard Protocol Items: Recommendations for 15 Interventional Trials (SPIRIT) guidelines ${ }^{40}$. The SPIRIT checklist is found in additional file 2. 
2 This trial is the first and currently sole ongoing trial to our knowledge to investigate topical sirolimus

3 in mucosal LM, namely LMLMs. LMLMs are a rare disease, and patients often carry a heavy

4 symptomatic burden, even with smaller-sized malformations. In particular, even with mild superficial

5 lesions, the aesthetic component might be of significant importance for young patients of school age.

6 There are currently no other therapeutic options for LMLMs than surgery or interventional procedures

7 such as laser or radiofrequency ablation, which are all painful procedures that carry the risk of

8 performing general anesthesia in children and often yield incomplete results with a significant rate of

9 local relapses. Moreover, surgery may be debilitating in the oral cavity in children, and laser or

10 radiofrequency ablation, although showing temporary efficacy, may hinder further chances of

11 complete surgical excision by inducing deep-tissue fibrosis ${ }^{11}$.

12 Thus, there is an unmet need for an easily carriable medical treatment with few side effects. Such a

13 treatment could be used as a first-intent monotherapy for smaller LMLMs or before a further planned

14 surgical excision in the watchful-waiting approach to more severe LMs.

15 Sirolimus has already shown efficacy as a systemic therapy for LM and carries interesting promise as a

16 cutaneous topical treatment. Despite its high molecular weight, 914.17 Da, which may hinder

17 transcutaneous diffusion, relevant clinical results with varying formulations could be achieved, with

18 concentrations ranging from $0.015 \%$ to $8 \%{ }^{22,31}$. From previous pharmacokinetic studies ${ }^{34}$ and case

19 series in oral inflammatory diseases ${ }^{27,28}$, we believe that therapeutic-range local concentrations can be 
1 obtained with daily application of topical sirolimus on the tongue, with clinical improvement as a

2 result, although such an intervention has yet to be reported in LM.

3 Given both the rarity of LMLM and the novelty of topical sirolimus, we planned the TOPGUN trial to 4 assess the safety and efficacy of topical sirolimus in LMLMs.

5 Despite the low recruitment capacity, we wanted this trial to be able to produce good-quality,

6 randomized evidence ${ }^{41}$. The individually randomized stepped-wedge design allows for using the

7 patient as their own control and to perform repeated outcome measurements to boost statistical power,

8 even in the absence of data regarding a carry-over effect after treatment discontinuation.

9 Also, we ensured a significant emphasis on trial acceptability. Patients under watchful waiting in our

10 tertiary center usually undergo a once- or twice-a-year follow-up, and they may come from several

11 dozens or hundreds of kilometers away. Hence, we designed a trial that minimizes on-the-spot study

12 center visits while maintaining both the required close clinical and biological surveillance and outcome

13 assessor blinding (by resorting to an external adjudication committee). Similarly, the stepped-wedge

14 design allows every patient to benefit from the experimental treatment regimen.

15 Indeed, although safety data in the literature is reassuring, notably the very low sirolimus systemic 16 passage, data on the exact setting of topical application in LMLMs are lacking. Safety procedures were 17 then designed for a "worst-case scenario" of a total sirolimus systemic diffusion, which would amount 18 to an oral 0.5 - to 1 -mg per day oral sirolimus regimen. We also gathered a DSMB in that respect. 
1 In the end, if the TOPGUN trial demonstrates a relevant clinical benefit and a good safety record, it

2 will be supported by good-quality evidence, and could pave the way for a rapid change in first-line

3 clinical practices in LMLMs.

\section{Trial Status}

5 The current version of the protocol is v3.0, dated $8^{\text {th }}$ December 2020. The first inclusion occurred on

$614^{\text {th }}$ January 2020, with a due recruitment period of 60 months (i.e. ends $14^{\text {th }}$ January 2025).

7 Five patients have been included, with two of them having completed the study.

\section{Declarations}

1. Ethics approval and consent to participate.

10 This protocol has been granted both ANSM (French Regulatory Authority) and CPP (Comité de

11 Protection des Personnes, French Ethical Review Committee) approval (19.03.28.46025). French law

12 does not require local ethics committee approval for each participating center.

13 The patient will be orally informed of the aim of the protocol and its procedures. Patients will also

14 receive a written information document. The written and informed consent of the patient, if obtained,

15 must be dated and signed both by the patient (or their parent or legal representatives). and the

16 investigator before any further study intervention. Children $\geq 16$ years old's consent will also be

17 sought. 
2. Consent for publication.

2 We will obtain patient's (or their parents' or legal representatives' if under 18) consent for publication

3 of the dataset and clinical photographs on trial enrollment. Children $\geq 16$ years old's consent will also

4 be sought.

5

3. Availability of data and materials

6 The datasets used and/or analyzed during the current study are available from the corresponding author

7 on reasonable request.

8

4. Competing interests

9 The authors declare that they have no competing interests.

10

5. Sponsor

11 The sponsor is the University Hospital Center of Tours, represented by Mr Julien Le Bonniec, Director 12 of Research and innovation, 2 Bd Tonnellé - 37044 Tours Cedex 9, Phone: +33 24747 70 07, Email:

13 j.lebonniec@chu-tours.fr

14

6. Funding 
1 This study is thoroughly funded thanks to an internal research tender organized by the University

2 Hospital Center of Tours in 2018 (“Appel d'Offre Interne 2018 - AOI2018). Copy of the original

3 funding document is found in additional file 3. No industry funding is involved.

4

9 We are indebted to Mr Wiebe de Jong from the Délégation à la Recherche Clinique et à l'Innovation,

10 CHRU Tours, 37000, Tours, France, Mrs Michèle Carriot and Frédérique Musset, Clinical

11 Investigation Center, INSERM 1415, CHRU Tours, 37000, Tours, France and Mrs Emiliène A.Edee,

12 from the Department of Dermatology and Reference Center for Rare Diseases and Vascular

13 Malformations (MAGEC),

14 We also deeply thank Dr Alice Phan (Department of Dermatology, Hospital University Centre of 15 Lyon, France), Dr Claire Abasq (Department of Dermatology, Hospital University Centre of Nantes, 16 France and Prof Mahtab Samimi (Department Of Dermatology, Hospital University Centre of Tours, 17 France) for participating in the adjudication committee for the primary outcome and Dr Bénédicte 18 Lebrun-Vignes, Pharmacologist, Department of Pharmacology (AP-HP, Hospital Pitié-Salpêtrière, 19 France), Dr Catherine Droitcourt, Dermatologist, Department Of Dermatology, (University Hospital of 
1 Rennes, France) and Dr Stéphanie Mallet, Dermatologist, Department Of Dermatology, (University

2 Hospital of Marseille, France) for being part of the Data Safety and Monitoring Board.

\section{Additional files}

4 Copy of the ethics committee agreement is found in additional file 1 (Additional_file_1.pdf)

5 The SPIRIT checklist is found in additional file 2 (Additional_file_2.docx)

6 Copy of the original funding document is found in additional file 3 (Additional_file_3.pdf)

\section{$7 \quad$ Figures}

8 Figure 1: Study Diagram (separate file: Figure_1.pdf)

9 


\section{$1 \quad$ Tables}

2 Table 1 Participant Timeline (group 1: treatment introduction at week 0)

3

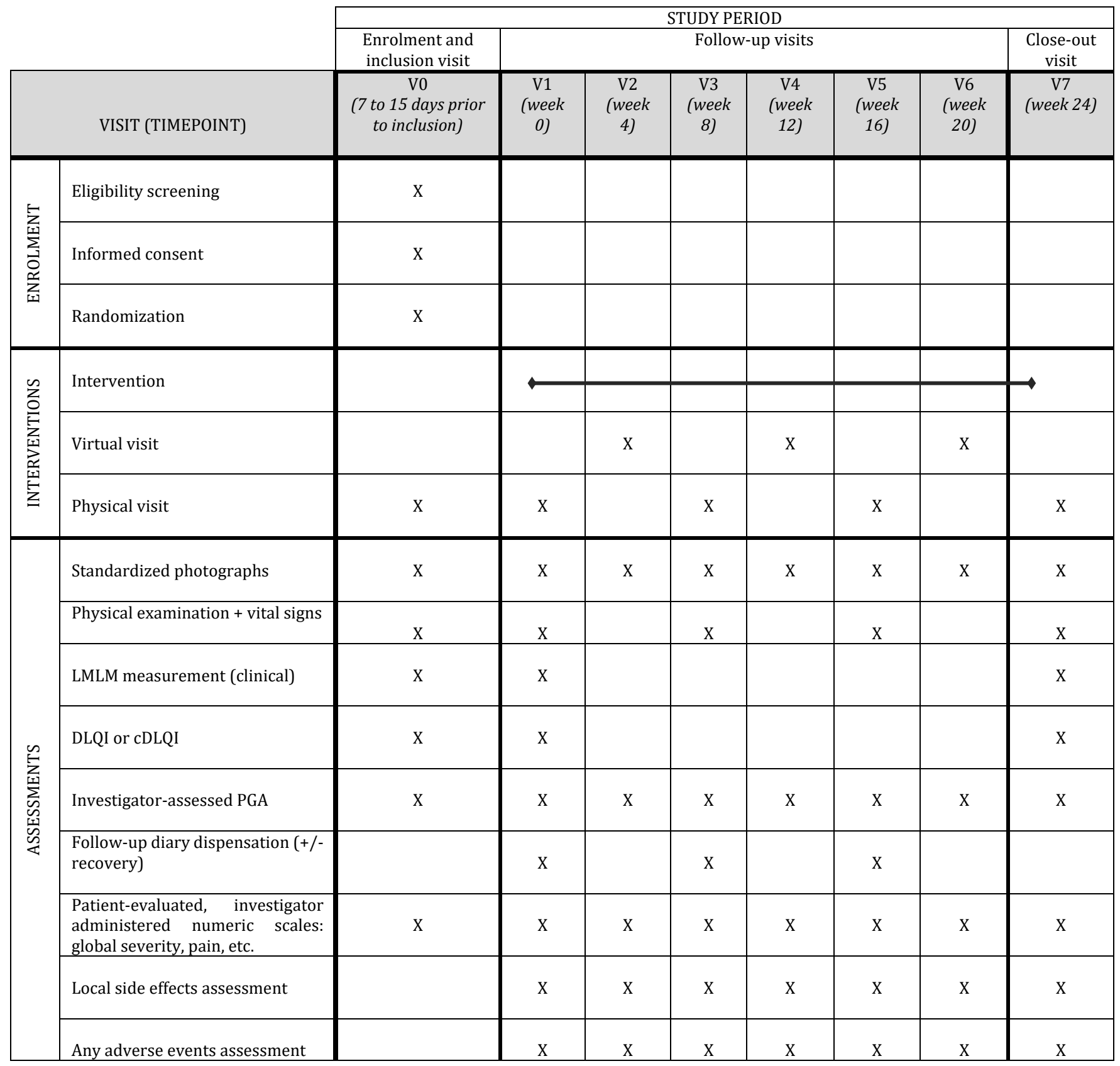




\begin{tabular}{|c|c|c|c|c|c|c|c|c|}
\hline Residual sirolimus dosing & & & $\mathrm{X}$ & $\mathrm{X}$ & & $\mathrm{X}$ & & $\mathrm{X}$ \\
\hline $\begin{array}{l}\text { Safety blood testing (CBC, LF, KF, } \\
\text { glycaemia, lipids) }\end{array}$ & $\mathrm{X}$ & & & $\mathrm{x}$ & & $\mathrm{X}$ & & $\mathrm{X}$ \\
\hline Urinary pregnancy test* & $\mathrm{X}$ & $\mathrm{X}$ & $\mathrm{X}$ & $\mathrm{X}$ & $\mathrm{X}$ & $\mathrm{X}$ & $\mathrm{X}$ & $\mathrm{X}$ \\
\hline Drug dispensation** & & $8 w$ & & $8 w$ & & $8 w$ & & \\
\hline
\end{tabular}

* For women of childbearing potential, until 3 months after study close-up.

** Drug dispensation: 3 bottles for 8 weeks (8w) or 2 bottles for 4 weeks (4w)

5 Table 2 Participant Timeline (group 2: treatment introduction at week 4)

6

\begin{tabular}{|c|c|c|c|c|c|c|c|c|c|}
\hline & \multicolumn{8}{|c|}{ STUDY PERIOD } \\
\hline & & Enrolment and & & & Follov & p visits & & & Close-out \\
\hline \multicolumn{2}{|r|}{ VISIT (TIMEPOINT) } & $\begin{array}{c}\mathrm{V} 0 \\
\text { (7 to } 15 \text { days prior } \\
\text { to inclusion) }\end{array}$ & $\begin{array}{c}\mathrm{V} 1 \\
\text { (week } \\
0)\end{array}$ & $\begin{array}{l}\mathrm{V} 2 \\
\text { (week } \\
4)\end{array}$ & $\begin{array}{l}\mathrm{V} 3 \\
\text { (week } \\
8 \text { ) }\end{array}$ & $\begin{array}{c}\mathrm{V} 4 \\
\text { (week } \\
12 \text { ) }\end{array}$ & $\begin{array}{c}\mathrm{V} 5 \\
\text { (week } \\
16)\end{array}$ & $\begin{array}{l}\mathrm{V} 6 \\
\text { (week } \\
20)\end{array}$ & $\begin{array}{c}\mathrm{V7} \\
\text { (week 24) }\end{array}$ \\
\hline \multirow{3}{*}{ 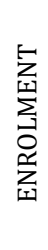 } & Eligibility screening & $\mathrm{X}$ & & & & & & & \\
\hline & Informed consent & $\mathrm{x}$ & & & & & & & \\
\hline & Randomization & $\mathrm{x}$ & & & & & & & \\
\hline \multirow{3}{*}{ 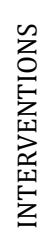 } & Intervention & & & & & & & & \\
\hline & Virtual visit & & $\mathrm{X}$ & & $\mathrm{X}$ & & $\mathrm{X}$ & & \\
\hline & Physical visit & $\mathrm{X}$ & & $\mathrm{x}$ & & $\mathrm{X}$ & & $\mathrm{x}$ & $\mathrm{X}$ \\
\hline \multirow{4}{*}{ 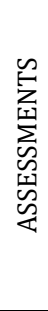 } & Standardized photographs & $\mathrm{X}$ & $\mathrm{X}$ & $\mathrm{X}$ & $\mathrm{X}$ & $\mathrm{x}$ & $\mathrm{X}$ & $\mathrm{x}$ & $\mathrm{X}$ \\
\hline & Physical examination + vital signs & $\mathrm{X}$ & & $\mathrm{X}$ & & $\mathrm{X}$ & & $\mathrm{X}$ & $\mathrm{X}$ \\
\hline & LMLM measurement (clinical) & $\mathrm{x}$ & & $\mathrm{x}$ & & & & & $\mathrm{X}$ \\
\hline & DLQI or cDLQI & $\mathrm{X}$ & & $\mathrm{X}$ & & & & & $\mathrm{X}$ \\
\hline
\end{tabular}




\begin{tabular}{|c|c|c|c|c|c|c|c|c|}
\hline Investigator-assessed PGA & $\mathrm{X}$ & $\mathrm{X}$ & $\mathrm{X}$ & $\mathrm{X}$ & $\mathrm{X}$ & $\mathrm{X}$ & $\mathrm{X}$ & $\mathrm{X}$ \\
\hline $\begin{array}{l}\text { Patient-evaluated, investigator } \\
\text { administered numeric scales: } \\
\text { global severity, pain, etc. }\end{array}$ & $\mathrm{X}$ & $\mathrm{X}$ & $\mathrm{X}$ & $\mathrm{X}$ & $\mathrm{X}$ & $\mathrm{X}$ & $\mathrm{X}$ & $\mathrm{X}$ \\
\hline Local side effects assessment & & $\mathrm{X}$ & $\mathrm{X}$ & $\mathrm{X}$ & $\mathrm{X}$ & $\mathrm{X}$ & $\mathrm{X}$ & $\mathrm{X}$ \\
\hline Any adverse events assessment & & $\mathrm{X}$ & $\mathrm{X}$ & $\mathrm{X}$ & $\mathrm{X}$ & $\mathrm{X}$ & $\mathrm{X}$ & $\mathrm{X}$ \\
\hline Residual sirolimus dosing & & & & $\mathrm{X}$ & $\mathrm{X}$ & & $\mathrm{X}$ & $\mathrm{X}$ \\
\hline $\begin{array}{l}\text { Safety blood testing (CBC, LF, KF, } \\
\text { glycaemia, lipids) }\end{array}$ & $\mathrm{X}$ & & & & $\mathrm{X}$ & & $\mathrm{X}$ & $\mathrm{X}$ \\
\hline Urinary pregnancy test* & $\mathrm{X}$ & $\mathrm{X}$ & $\mathrm{X}$ & $\mathrm{X}$ & $\mathrm{X}$ & $\mathrm{X}$ & $\mathrm{X}$ & $\mathrm{X}$ \\
\hline Drug dispensation** & & & $8 w$ & & $8 w$ & & $4 w$ & \\
\hline
\end{tabular}

3 * For women of childbearing potential, until 3 months after study close-up. 
1 Table 3 Participant Timeline (group 3: treatment introduction at week 8)

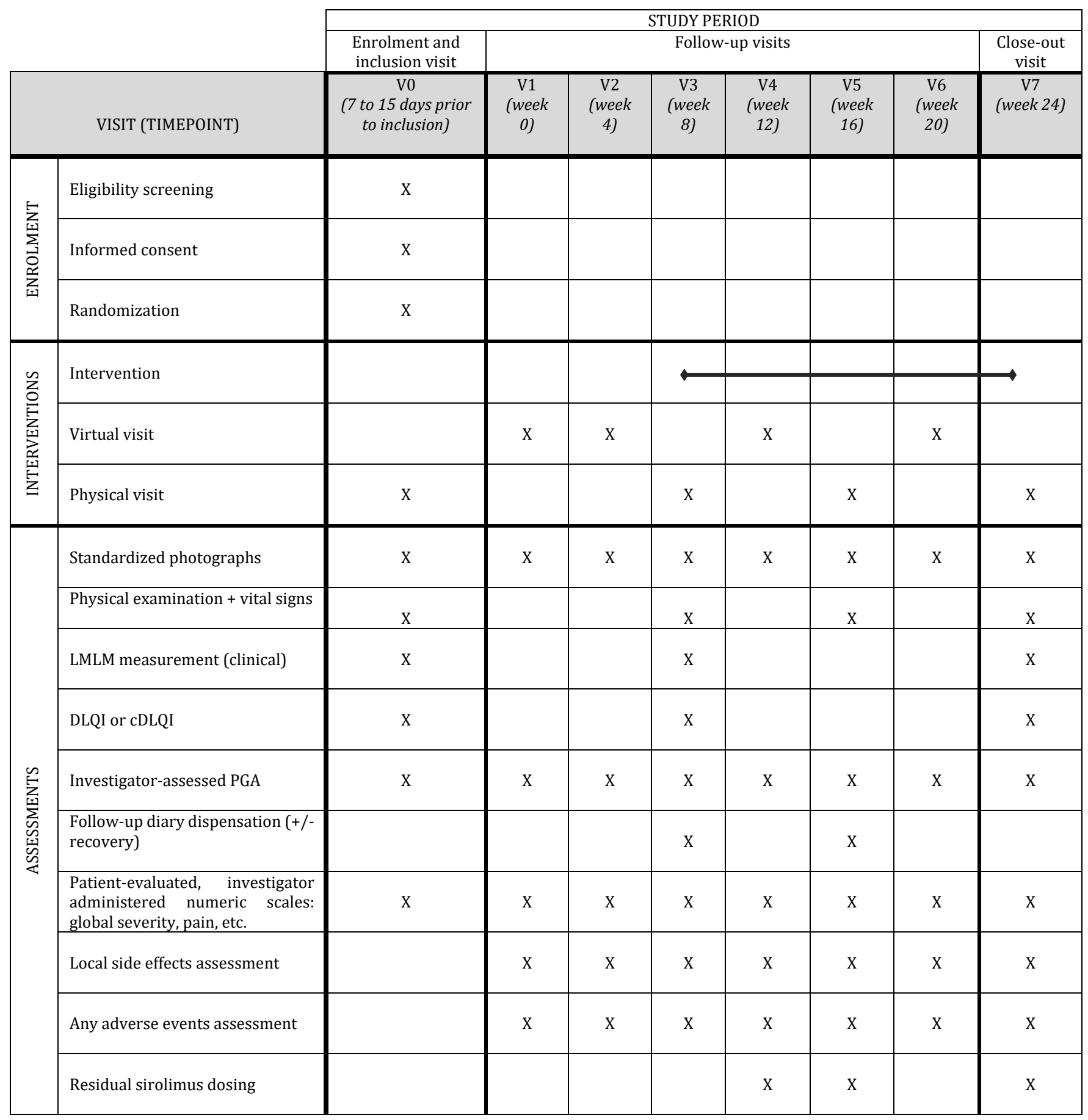




\begin{tabular}{|c|c|c|c|c|c|c|c|c|}
\hline $\begin{array}{l}\text { Safety blood testing (CBC, LF, KF, } \\
\text { glycaemia, lipids) }\end{array}$ & $\mathrm{X}$ & & & & & $\mathrm{X}$ & & $\mathrm{X}$ \\
\hline Urinary pregnancy test* & $\mathrm{X}$ & $\mathrm{X}$ & $\mathrm{X}$ & $\mathrm{X}$ & $\mathrm{X}$ & $\mathrm{X}$ & $\mathrm{X}$ & $\mathrm{X}$ \\
\hline Drug dispensation** & & & & $8 w$ & & $8 w$ & & \\
\hline
\end{tabular}

* For women of childbearing potential, until 3 months after study close-up.

** Drug dispensation: 3 bottles for 8 weeks (8w) or 2 bottles for 4 weeks (4w)

5 Table 4 Participant Timeline (group 4: treatment introduction at week 12)

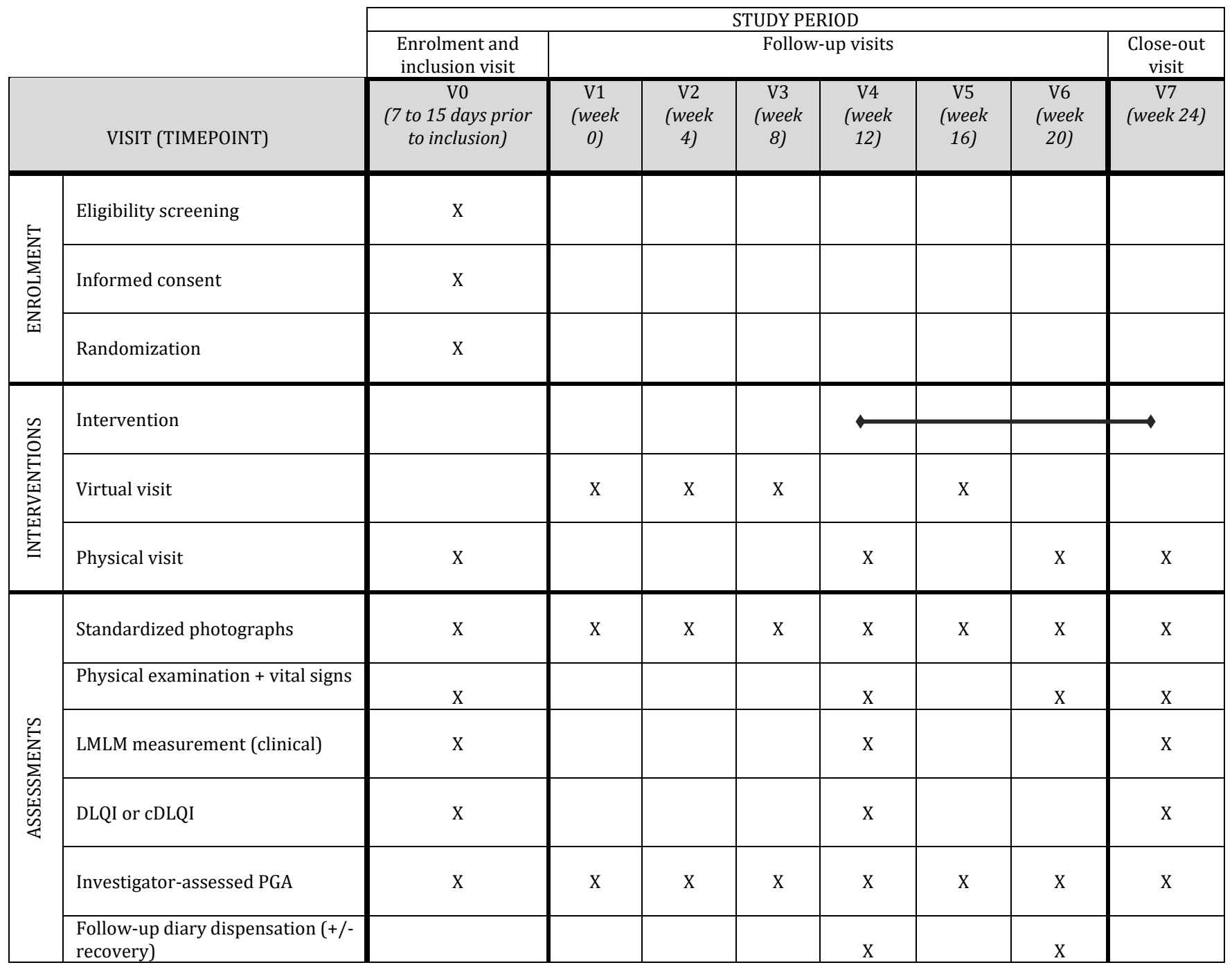




\begin{tabular}{|c|c|c|c|c|c|c|c|c|}
\hline & & & & & & & & \\
\hline $\begin{array}{l}\text { Patient-evaluated, investigator } \\
\text { administered numeric scales: } \\
\text { global severity, pain, etc. }\end{array}$ & $X$ & $X$ & $X$ & $X$ & $X$ & $X$ & $X$ & $\mathrm{X}$ \\
\hline Local side effects assessment & & $\mathrm{X}$ & $\mathrm{X}$ & $\mathrm{x}$ & $\mathrm{X}$ & $\mathrm{x}$ & $\mathrm{X}$ & $\mathrm{X}$ \\
\hline Any adverse events assessment & & $\mathrm{X}$ & $\mathrm{X}$ & $\mathrm{x}$ & $\mathrm{X}$ & $\mathrm{x}$ & $\mathrm{X}$ & $\mathrm{X}$ \\
\hline Residual sirolimus dosing & & & & & & $\mathrm{X}$ & $\mathrm{X}$ & $\mathrm{X}$ \\
\hline $\begin{array}{l}\text { Safety blood testing (CBC, LF, KF, } \\
\text { glycaemia, lipids) }\end{array}$ & X & & & & & & $X$ & $X$ \\
\hline Urinary pregnancy test* & $\mathrm{X}$ & $\mathrm{X}$ & $\mathrm{X}$ & $\mathrm{X}$ & $\mathrm{X}$ & $\mathrm{X}$ & $\mathrm{X}$ & $\mathrm{X}$ \\
\hline Drug dispensation** & & & & & $8 w$ & & $4 w$ & \\
\hline
\end{tabular}

2 * For women of childbearing potential, until 3 months after study close-up.

$3 \quad * *$ Drug dispensation: 3 bottles for 8 weeks (8w) or 2 bottles for 4 weeks (4w)

\section{References}

6 1. Renton JP, Smith RJH. Current treatment paradigms in the management of lymphatic malformations. The Laryngoscope. 2011;121(1):56-59. doi:10.1002/lary.20768

2. ISSVA Classification of Vascular Anomalies (2018 International Society for the Study of

9 Vascular Anomalies Available at "issva.org/classification"; Accessed November 2020

3. Wassef M, Blei F, Adams D, et al. Vascular Anomalies Classification: Recommendations from the

11 International Society for the Study of Vascular Anomalies. PEDIATRICS. 2015;136(1):e203-e214. doi:10.1542/peds.2014-3673 
4. Wiegand S, Eivazi B, Barth PJ, et al. Pathogenesis of lymphangiomas. Virchows Arch. 2008;453(1):1-8. doi:10.1007/s00428-008-0611-z

5. Osborn AJ, Dickie P, Neilson DE, et al. Activating PIK3CA alleles and lymphangiogenic phenotype of lymphatic endothelial cells isolated from lymphatic malformations. Human Molecular Genetics. 2015;24(4):926-938. doi:10.1093/hmg/ddu505

6. Hammill AM, Wentzel M, Gupta A, et al. Sirolimus for the treatment of complicated vascular anomalies in children. Pediatr Blood Cancer. 2011;57(6):1018-1024. doi:10.1002/pbc.23124

7. Groupe de Recherche de la Société Française de Dermatologie Pédiatrique, Maruani A, Boccara O,

10. Elluru RG, Balakrishnan K, Padua HM. Lymphatic malformations: Diagnosis and management.

8. Groupe de Recherche de la Société Française de Dermatologie Pédiatrique, Leducq S, Caille A, et al. Topical sirolimus $0.1 \%$ for treating cutaneous microcystic lymphatic malformations in children and adults (TOPICAL): protocol for a multicenter phase 2, within-person, randomized, doubleblind, vehicle-controlled clinical trial. Trials. 2019;20(1):739. doi:10.1186/s13063-019-3767-8

9. de Serres LM, Sie KCY, Richardson MA. Lymphatic Malformations of the Head and Neck: A Proposal for Staging. Archives of Otolaryngology - Head and Neck Surgery. 1995;121(5):577-582. doi:10.1001/archotol.1995.01890050065012 Seminars in Pediatric Surgery. 2014;23(4):178-185. doi:10.1053/j.sempedsurg.2014.07.002 
1 11. Wiegand S, Eivazi B, Zimmermann AP, et al. Microcystic Lymphatic Malformations of the

2 Tongue: Diagnosis, Classification, and Treatment. Arch Otolaryngol Head Neck Surg. 2009;135(10):976. doi:10.1001/archoto.2009.131

4 12. Wittekindt C, Michel O, Streppel M, et al. Lymphatic malformations of the head and neck:

5 Introduction of a disease score for children, Cologne Disease Score (CDS). International Journal of Pediatric Otorhinolaryngology. 2006;70(7):1205-1212. doi:10.1016/j.ijporl.2005.12.013

13. Adams DM, Trenor CC, Hammill AM, et al. Efficacy and Safety of Sirolimus in the Treatment of Complicated Vascular Anomalies. PEDIATRICS. 2016;137(2):e20153257-e20153257. doi:10.1542/peds.2015-3257

10 14. Perkins JA, Manning SC, Tempero RM, et al. Lymphatic malformations: Review of current 11 treatment. Otolaryngol Head Neck Surg. 2010;142(6):795-803.e1. doi:10.1016/j.otohns.2010.02.026

13 15. Bajaj Y, Hewitt R, Ifeacho S, Hartley BEJ. Surgical excision as primary treatment modality for

16. Defnet AM, Bagrodia N, Hernandez SL, Gwilliam N, Kandel JJ. Pediatric lymphatic malformations: evolving understanding and therapeutic options. Pediatr Surg Int. 2016;32(5):425-

17. Lamming DW. Inhibition of the Mechanistic Target of Rapamycin (mTOR)-Rapamycin and Beyond. Cold Spring Harb Perspect Med. 2016;6(5):a025924. doi:10.1101/cshperspect.a025924 
1 18. Kobayashi S, Kishimoto T, Kamata S, Otsuka M, Miyazaki M, Ishikura H. Rapamycin, a specific inhibitor of the mammalian target of rapamycin, suppresses lymphangiogenesis and lymphatic metastasis. Cancer Science. 2007;98(5):726-733. doi:10.1111/j.1349-7006.2007.00439.x

19. Boscolo E, Coma S, Luks VL, et al. AKT hyper-phosphorylation associated with PI3K mutations in lymphatic endothelial cells from a patient with lymphatic malformation. Angiogenesis. 2015;18(2):151-162. doi:10.1007/s10456-014-9453-2

20. Fereydooni A, Dardik A, Nassiri N. Molecular changes associated with vascular malformations. Journal of Vascular Surgery. 2019;70(1):314-326.e1. doi:10.1016/j.jvs.2018.12.033

21. Paghdal KV, Schwartz RA. Sirolimus (rapamycin): From the soil of Easter Island to a bright future. Journal of the American Academy of Dermatology. 2007;57(6):1046-1050. doi:10.1016/j.jaad.2007.05.021

22. Fogel AL, Hill S, Teng JMC. Advances in the therapeutic use of mammalian target of rapamycin (mTOR) inhibitors in dermatology. Journal of the American Academy of Dermatology. 2015;72(5):879-889. doi:10.1016/j.jaad.2015.01.014

23. Marsh DJ, Trahair TN, Martin JL, et al. Rapamycin treatment for a child with germline PTEN mutation. Nat Rev Clin Oncol. 2008;5(6):357-361. doi:10.1038/ncponc1112

24. Nadal M, Giraudeau B, Tavernier E, Jonville-Bera A, Lorette G, Maruani A. Efficacy and Safety of Mammalian Target of Rapamycin Inhibitors in Vascular Anomalies: A Systematic Review. Acta Derm Venerol. 2016;96(4):448-452. doi:10.2340/00015555-2300

25. clinicaltrials.gov; Accessed November 2020 
26. Ormerod AD, Shah SAA, Copeland P, Omar G, Winfield A. Treatment of psoriasis with topical sirolimus: preclinical development and a randomized, double-blind trial. Br J Dermatol. 2005;152(4):758-764. doi:10.1111/j.1365-2133.2005.06438.x

27. Soria A, Agbo-Godeau S, Ta\&iuml;eb A, Franc\&egrave;s C. Treatment of Refractory Oral Erosive Lichen Planus with Topical Rapamycin: 7 Cases. Dermatology. 2009;218(1):22-25. doi:10.1159/000172830

28. Poot AM, Jonkman MF. Topical sirolimus for oral pemphigus vulgaris: 3 unresponsive cases. Journal of the American Academy of Dermatology. 2012;67(5):e228-e229. doi:10.1016/j.jaad.2012.04.032

29. Amin S, Lux A, Khan A, O’Callaghan F. Sirolimus Ointment for Facial Angiofibromas in Individuals with Tuberous Sclerosis Complex. International Scholarly Research Notices. 2017;2017:1-6. doi:10.1155/2017/8404378

30. Malissen N, Vergely L, Simon M, Roubertie A, Malinge M-C, Bessis D. Long-term treatment of cutaneous manifestations of tuberous sclerosis complex with topical $1 \%$ sirolimus cream: A prospective study of 25 patients. Journal of the American Academy of Dermatology. 2017;77(3):464-472.e3. doi:10.1016/j.jaad.2017.04.005

31. Leducq S, Giraudeau B, Tavernier E, Maruani A. Topical use of mammalian target of rapamycin inhibitors in dermatology: A systematic review with meta-analysis. Journal of the American Academy of Dermatology. 2019;80(3):735-742. doi:10.1016/j.jaad.2018.10.070 
32. Ivars M, Redondo P. Efficacy of Topical Sirolimus (Rapamycin) for the Treatment of Microcystic Lymphatic Malformations. JAMA Dermatol. 2017;153(1):103. doi:10.1001/jamadermatol.2016.3697

33. García-Montero P, del Boz J, Sanchez-Martínez M, Escudero Santos IM, Baselga E. Microcystic Lymphatic Malformation Successfully Treated With Topical Rapamycin. Pediatrics. 2017;139(5):e20162105. doi:10.1542/peds.2016-2105

34. Nudelman Z, Friedman M, Barasch D, et al. Levels of sirolimus in saliva and blood following (1) mouthwash application. Oral Dis. Published online March 2014:n/a-n/a. doi:10.1111/odi.12229

35. Cornu C, Kassai B, Fisch R, et al. Experimental designs for small randomised clinical trials: an algorithm for choice. Orphanet J Rare Dis. 2013;8(1):48. doi:10.1186/1750-1172-8-48

1136 . Hooper R, Knowles C. Improving the efficiency of individually randomized clinical trials by 12 staggering the introduction of the intervention. Statistics in Medicine. 2019;38(1):44-52. doi:10.1002/sim.7959

37. Lewis-Jones MS, Finlay AY. The Children's Dermatology Life Quality Index (CDLQI): initial validation and practical use. British Journal of Dermatology. 2010;132(6):942-949. doi:10.1111/j.1365-2133.1995.tb16953.x

38. Garduno J, Bhosle MJ, Balkrishnan R, Feldman SR. Measures used in specifying psoriasis lesion(s), global disease and quality of life: A systematic review. Journal of Dermatological Treatment. 2007;18(4):223-242. doi:10.1080/09546630701271807 
1 39. Tabolli S, Sampogna F, Pagliarello C, Paradisi A, Spagnoli A, Abeni D. Disease severity 2 evaluation among dermatological out-patients: a comparison between the assessments of patients 3 and physicians: Patient-physician disease severity evaluation: a comparison. Journal of the 4 European Academy of Dermatology and Venereology. 2012;26(2):213-218. doi:10.1111/j.1468$5 \quad 3083.2011 .04038 . x$

6 40. Chan A-W, Tetzlaff JM, Altman DG, et al. SPIRIT 2013 Statement: Defining Standard Protocol 7 Items for Clinical Trials. Ann Intern Med. 2013;158(3):200. doi:10.7326/0003-4819-158-3$8 \quad 201302050-00583$

9 41. Chalmers T. Randomize the First Patient! N Engl J Med. 1977;296(2):107-107.

10 doi:10.1056/NEJM197701132960214 
Figures

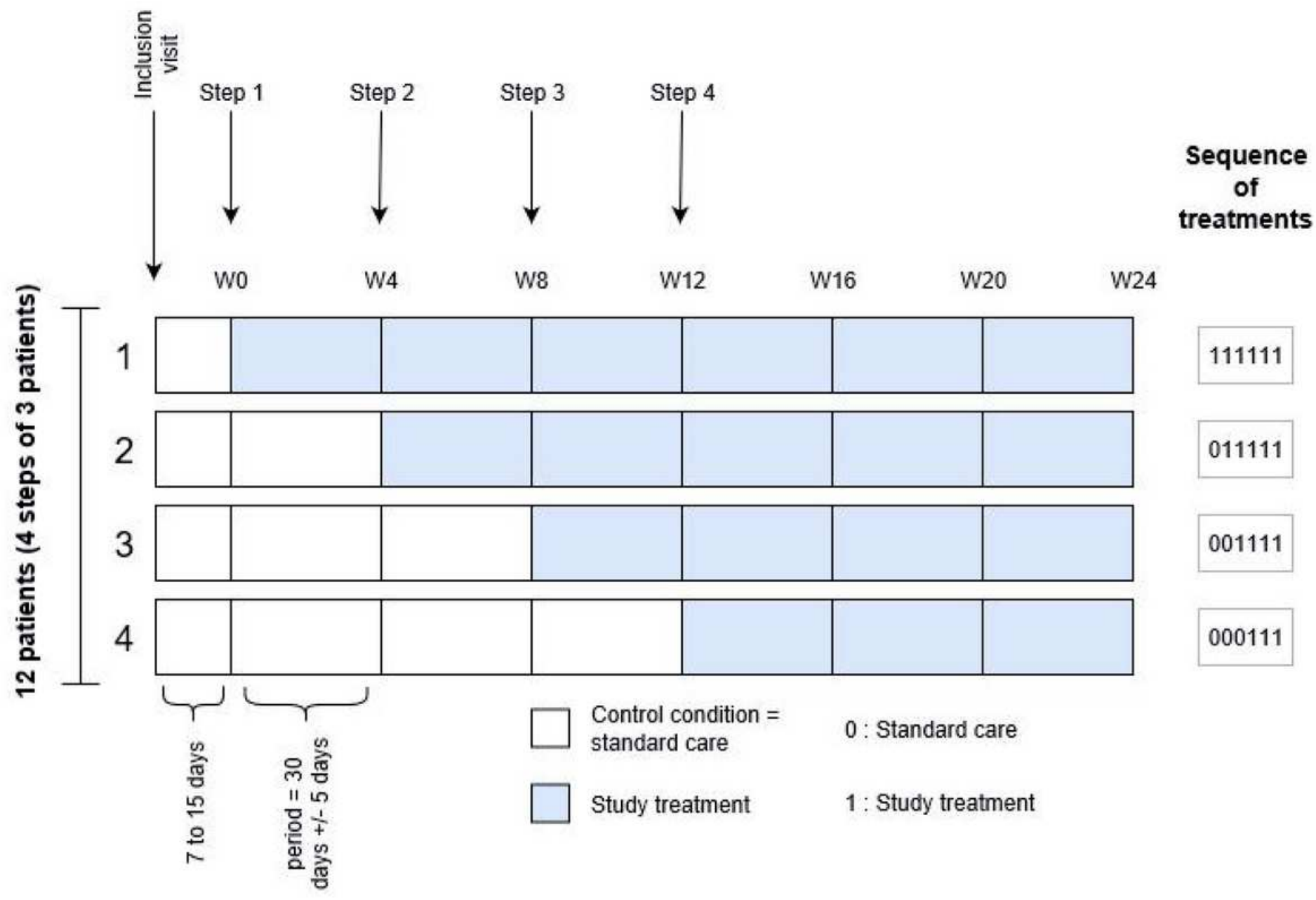

Figure 1

Study Diagram

\section{Supplementary Files}

This is a list of supplementary files associated with this preprint. Click to download.

- Additionalfile1.pdf

- Additionalfile2.docx

- Additionalfile3.pdf 